\title{
Cosic's Resonance Recognition Model for Protein Sequences and Photon Emission Differentiates Lethal and Non-Lethal Ebola Strains: Implications for Treatment
}

\author{
Nirosha J. Murugan, Lukasz M. Karbowski, Michael A. Persinger \\ Bioquantum Laboratory, Biomolecular Sciences and Behavioural Neuroscience Programs, Laurentian University, \\ Sudbury, Canada \\ Email: mpersinger@laurentian.ca
}

Received 7 October 2014; revised 4 November 2014; accepted 1 December 2014

Copyright (C) 2015 by authors and Scientific Research Publishing Inc.

This work is licensed under the Creative Commons Attribution International License (CC BY). http://creativecommons.org/licenses/by/4.0/

(c) (i) Open Access

\section{Abstract}

The Cosic Resonance Recognition Model (RRM) for amino acid sequences was applied to the classes of proteins displayed by four strains (Sudan, Zaire, Reston, Ivory Coast) of Ebola virus that produced either high or minimal numbers of human fatalities. The results clearly differentiated highly lethal and non-lethal strains. Solutions for the two lethal strains exhibited near ultraviolet $(\sim 230 \mathrm{~nm})$ photon values while the two asymptomatic forms displayed near infrared $(\sim 1000 \mathrm{~nm})$ values. Cross-correlations of spectral densities of the RRM values of the different classes of proteins associated with the genome of the viruses supported this dichotomy. The strongest coefficient occurred only between Sudan-Zaire strains but not for any of the other pairs of strains for SGP, the small glycoprotein that intercalated with the plasma cell membrane to promote insertion of viral contents into cellular space. A surprising, statistically significant cross-spectral correlation occurred between the "spike" glycoprotein component (GP1) of the virus that associated the anchoring of the virus to the mammalian cell plasma membrane and the Schumann resonance of the earth whose intensities were determined by the incidence of equatorial thunderstorms. Previous applications of the RRM to shifting photon wavelengths emitted by melanoma cells adapting to reduced ambient temperature have validated Cosic's model and have demonstrated very narrowwave-length (about $10 \mathrm{~nm}$ ) specificity. One possible ancillary and non-invasive treatment of people within which the fatal Ebola strains are residing would be whole body application of narrow band near-infrared light pulsed as specific physiologically-patterned sequences with sufficient radiant flux density to perfuse the entire body volume.

\section{Keywords}

Cosic Resonance Recognition Model, Ebola Virus, Fatal vs Asymptomatic Forms, Ultraviolet vs 


\section{Introduction}

From a biophysical and ecological perspective, the proliferation and density of all life forms, including the human population, are subject to physical constraints determined by the parameters of physical and chemical reactions within the terrestrial environment. The intrinsic processes often described as dynamic equilibrium suggest there are mechanisms that mediate this control. Minute alterations in the genetic expression of opportunistic infections or modified vulnerability to pathogens have been considered as the standard forms of contagion by which populations are controlled or eliminated. However interpretations are subject to change, such as for the case of malaria that was once attributed to "bad air" before the recondite stimuli responsible for this disease was measured, and often require a significant change in perspective from contemporary assumptions. Here we present an alternative mechanism for the proliferation of Ebola, the possible biophysical mechanism for the marked strain variation in fatality, the potential etiology, and a possible non-invasive treatment.

The current Zaire Ebola virus is a subset of the genus of Ebola viruses for which the most typical symptom is fatal hemorrhagic fever in human beings. The recent (2014) proliferation in Africa is considered similar if not identical to that form first identified in the Democratic Republic of Congo and is considered similar to the Marburg virus. Transmission is presumed to involve proximity with fluids originating from an infected person. However, unlike the first known manifestations 1976, the proliferation has escalated since the Spring of 2014 although precise inflections of the growth curves for prevalence and incidence could extend to 2012.

Not all subsets of Ebola are deadly. There are at least four locations where the manifestations occurred (year of onset in parentheses). Two of them, Reston (1995) and Ivory Coast (1994) were associated with minimum or no mortality. The Sudan (1976) and Zaire (1976) varieties were associated with $54 \%$ and $88 \%$ mortality, respectively.

The Ebola virus contains $~ 19,000$ base pairs and encodes for seven structural proteins whose sequences have been isolated [1]. The essential structure is a cylinder or tube whose length ranges within the near infrared wavelength (800 to $1000 \mathrm{~nm}$ ) with a radius of $\sim 40 \mathrm{~nm}$ ( $251 \mathrm{~nm}$ circumference). From the bilayer lipid surface, glycoproteins extend as $10 \mathrm{~nm}$ projections with interspaces of $\sim 10 \mathrm{~nm}$ [2]. The latter is effectively the same width as a plasma membrane of a mammalian cell and the equivalence of the phase modulation for visible photon emissions $\left(\sim 10^{-19} \mathrm{~J}\right)$ from cells [3] resulting in energies of $\sim 10^{-20} \mathrm{~J}$. This increment of energy is associated with a plethora of critical biophysical processes that includes the sequestering of ligands to receptors and the resting membrane potential [4].

The virus itself has four strains with a genome of $19 \mathrm{kB}$. This genome encodes 8 - 9 proteins that facilitate infection and proliferation within the host organism. From the NIH (National Institute of Health) databank we obtained the genomic sequences for the four strains Sudan $(18,875)$, Zaire $(18,839)$, Reston $(18,960)$ and Ivory Coast $(18,930)$ as well as the associated (34) proteins from the various strains. The acronyms, names and number of amino acids for the major proteins are shown in Table 1.

Table 1. Acronyms, name and Amino Acid (AA) lengths of components of Ebola.

\begin{tabular}{ccc}
\hline Acronym & Protein & Amino Acids \\
\hline NP & Nucleoprotein & 738 \\
VP35 & Polymerase complex protein & 329 \\
VP40 & Matrix protein & 326 \\
GP1 & Spike glycoprotein & 676 \\
sGP & Small secreted glycoprotein & 372 \\
VP30 & Minor nucleoprotein & 288 \\
VP24 & Membrane-associated protein & 251 \\
L & RNA-dependent RNA polymerase & 2210 \\
\hline
\end{tabular}




\section{Irena Cosic's Resonant Recognition Model (RRM)}

The Resonant Recognition Model (RRM) was developed by Irene Cosic [5] who was attempting to reconcile the unexpected, marked resemblances between functionally dissimilar proteins. She assumed that a type of spectral density of the spatial sequences of the amino acids in different proteins might be more revealing than simply comparing classic chemical "structures". The model is based upon representing the protein's primary structure as numerical series by assigning each amino acid with a physical value. This value was the energy of delocalized electrons for each amino acid. She has obtained characteristic RRM values for different functional groups of proteins and DNA regulatory sequences.

We [6] have experimentally supported the predictions and applications of the Cosic model by measuring the photon emissions from mouse (B16) melanoma cells that had been removed from incubation. The cells emit specific increments of $10 \mathrm{~nm}$ wavelengths from the near ultraviolet through the visible to the near infrared range as measured by photomultiplier units. This shift in photon emission wavelengths (as inferred by the results of different filters) changed from primarily near infrared to near ultraviolet over a ten hour period. Specific chemical activators or inhibitors for specific wavelengths based upon the RRM elicited either enhancement or diminishment of photons at the specific wavelength predicted by Cosic. Activators or inhibitors predicted for other wavelengths were not effective or much less effective. The spike in near-infrared energies preceded a spike in near-ultraviolet energies by about 3 hours. The temporal sequence was consistent with the activation of signaling pathways (near-infrared) followed by activation of protein-structural factors (near-ultraviolet).

$\mathrm{Wu}$ and Persinger [7] had shown that the wavelength of infrared photons predicted from Cosic's model for cytochrome c and cytochrome oxidase II, proteins associated with activation in the regenerating blastema within planarian, facilitated the rate of growth of sectioned organisms. The power density of the $880 \mathrm{~nm}$ light was $\sim 10^{-3} \mathrm{~W} \cdot \mathrm{m}^{-2}$. The energy at the level of a symmetrical patch of plasma cell membrane $\left(10^{-16} \mathrm{~m}^{2}\right)$ would have been $\sim 10^{-19} \mathrm{~J}$. When considered together the potential utility of RRM for pursuing the optimal photon frequencies that could differentially affect viral activity was considered feasible.

Biophotons are emitted by bacteria [8] and cells [9] and may be a means by which intercellular communications [10] occur rather than just a spurious correlate of biochemical activity. Their power (flux) densities are in the order of $10^{-11}$ to $10^{-13} \mathrm{~W} \cdot \mathrm{m}^{-2}$. Biologically-relevant reactions such as the addition of hydrogen peroxide to hypochlorite solutions emit copious photons and may be involved with non-local interactions between chemical reactions [11] as well as shifts in $\mathrm{pH}$ [12]. Photon emissions from microtubual preparations respond to the application of relatively weak $(\mu \mathrm{T})$ extremely low frequency magnetic fields when they display changing angular velocities around a circular array of solenoids [13]. Comparable magnetic field strengths that match the "membrane magnetic moment" of cells facilitate the release of photons and suggest the involvement of very small energies such as the difference between electron spin and orbital magnetic moments [14]. At a cellular level biophoton emission is induced by heat shock [15].

Applying light with specific frequencies can preserve biological function. Exposure of optic nerves after partial injury to about $250 \mathrm{~W} \cdot \mathrm{m}^{-2}$ of $670 \mathrm{~nm}$ for $30 \mathrm{~min}$ reduced oxidative stress [16] and attenuated secondary degeneration. Low power laser light $(685 \mathrm{~nm})$ exposure for $3 \mathrm{~min}$ to $910 \mathrm{~W} \cdot \mathrm{m}^{-2}$ stimulated stem cell proliferation in planaria [17]. In fact near-infrared photoimmunotherapy that targets specific membrane molecules [18] has been successful in vivo by binding to the cell membrane which has been shown by Dotta et al. [19] to be a primary source of biophotons in the order of $10^{-20} \mathrm{~J}$ per s per reaction. Because visible light penetrates the mammalian brain and body the presence of encephalopsin (extraretinal opsins within for example brain tissue) suggests external photons of specific wavelengths may be more effective than now appreciated. Opsins mediate transmembrane proteins that act on G-protein-coupled receptors [20]. Exposure of the human skull (via the ear canal) to blue $(465 \mathrm{~nm})$ LEDs with a luminous flux density of about $10 \mathrm{~W} \cdot \mathrm{m}^{-2}$ elicits discernable changes throughout the brain as inferred by fMRI activity [21].

\section{Cosic Procedure}

The genomic and proteomic information for each of the four strains of the Ebola virus were obtained from the National Center for Biotechnology Information data (NCBI). The NCBI reference sequence or identification number for each strain, along with the initial year of outbreak and resulting deaths can be seen in Table 2. The NCBI reference (RefSeq) was: Zaire: http://www.ncbi.nlm.nih.gov/nuccore/10313991. The suffixes for the Sudan, Reston, and Ivory Coast references were: 55770807, 2278922, and 302315369, respectively. 
Table 2. Ebola Virus Strain, the NCBI RefSeq, Cosic's Resonant Recognition Model (RRM), the actual or true frequency, and the percentage of deaths of each strain.

\begin{tabular}{cccccc}
\hline Ebola Virus Strain & NCBI RefSeq & Year & RRM frequency & True Frequency & Deaths \\
\hline Sudan & NC_006432.1 & 1976 & 0.874578947 & $1.3 \mathrm{E}+15$ & $53 \%$ \\
Zaire & NC_002549.1 & 1976 & 0.875973684 & $1.31 \mathrm{E}+15$ & $88 \%$ \\
Reston & NC_004161.1 & 1995 & 0.1887494812 & $2.52 \mathrm{E}+14$ & $0 \%$ \\
Tai Forest (Cote d'Ivoire) & NC_014372.1 & 1994 & 0.1959063482 & $2.92 \mathrm{E}+14$ & $0 \%$ \\
\hline
\end{tabular}

The primary amino acid sequence was transformed into a numerical sequence using the Resonant Recognition Model (RRM). Each of the 20 amino acids in the entire sequence was assigned an electron-ion interaction potential (EIIP) value [4]. This value represents the average energy state of all of the valence electron associated with that amino acid. The numerical sequence was then subjected to a signal analysis to determine a characteristic RRM frequency. The RRM frequency was converted to a true frequency by determining the appropriate wavelength using the function $\mathrm{f}_{\mathrm{RRM}}=201 / \lambda$. This method was also applied to the genomic sequence of each strain, where each nucleotide was represented by an EIIP value, and then subjected to signal analysis.

\section{Results of Cosic's RRM}

As shown in Table 2, the results indicated a clear difference between the rarely fatal and very fatal strains of Ebola. The primary resonance frequency $\mathrm{f}_{\mathrm{RRM}}$ for the two deadliest strains (Sudan and Zaire) were 0.87457 and 0.8759 . This would be equivalent to an actual frequency of $1.3044 \times 10^{15}$ and $1.3065 \times 10^{15} \mathrm{~Hz}$, respectively. Although very similar the difference between the two strains is equivalent to $\sim 0.01 \mathrm{eV}\left(10^{-21} \mathrm{~J}\right)$ which is similar to the average energy required for A-T/C-G base pairing in the human genome.

Assuming the velocity of light in a vacuum, the equivalent wavelengths for the Cosic frequencies for the two stains would be $\sim 230 \mathrm{~nm}$. If we assume the variable velocity of light in water [22], which ranges from $2.147 \times$ $10^{8} \mathrm{~m} \cdot \mathrm{s}^{-1}$ for $370 \mathrm{~nm}$ to $2.206 \times 10^{8} \mathrm{~m} \cdot \mathrm{s}^{-1}$ at $520 \mathrm{~m}$, the wavelengths would be closer to $\sim 160$ to $165 \mathrm{~nm}$ depending upon inferences of linearity. In other words the electromagnetic equivalent of the Cosic frequency would involve photons within the near ultraviolet band. Interestingly, the radius of the circular wavelength (230 $\mathrm{nm}$ ) would be $36.6 \mathrm{~nm}$, that is, within the range of the radius of the Ebola virus.

On the other hand the least fatal Ebola strains, the Reston and Ivory Coast varieties, which after infecting the host are asymptomatic, display Cosic frequencies of $2.51691 \times 10^{14} \mathrm{~Hz}$ and $2.92195 \times 10^{14} \mathrm{~Hz}$, respectively. The equivalent wavelength for photons would be 1.19 and $1.03 \mu \mathrm{m}$, respectively, that is within the near infrared range. If we assume the adjustment for the velocity of light in water, for example, $2.3 \times 10^{8} \mathrm{~m} \cdot \mathrm{s}^{-1}$ the effective Cosic solution would be narrow-band wavelengths of 912 and $815 \mathrm{~nm}$. This is within the range of the length (800 to $1000 \mathrm{~nm}$ ) the virus.

There are major implications for this clear dichotomy in association with photon frequency between the more lethal and non-symptomatic forms of Ebola. Functional wavelengths that encompass near-UV are usually associated with growth and dynamic protein changes. Wavelengths involving near-IR are associated with general activation. The clear discrepancy of wavelengths between the lethal and nonlethal strains could be sufficient to allow therapeutic intervention by applied, narrow band light spectra. If the viral activities operate similarly to what was measured with melanoma cells, the application of the photon wavelengths must be within $10 \mathrm{~nm}$ of the predicted Cosic frequency or there would be no effect [4].

The most parsimonious intervention would be the whole body application of the 1.02 to $1.19 \mu \mathrm{m}$ (near IR) wavelength to patients who have contracted Sudan and Zaire strains. If, as our melanoma and planarian experiments imply [4] [5], the photon frequencies predicted by the Cosic RRM are the equivalent of the molecular structure or a type of "virtual" structure, the IR should produce a non-lethal representation within the viral proteins. If valid, this could reduce the fatality by directly disrupting the intrinsic proliferative mechanisms. It would be essential to employ LED (Light Emitting Diodes) that were manufactured specifically for those frequencies. "Red" lights from incandescent sources or simply painted light bulbs based upon full (visible) spectrum emission would be less effective.

The optimal power or photon flux density of the near IR LED frequencies for whole body exposure may be 
less intense than anticipated. For treatment of SAD (Seasonal Affective Depression) white light in excess of 2500 lux or $\sim 1 \mathrm{~W} \cdot \mathrm{m}^{-2}$ (1 lux $\left.=\sim 1.5 \times 10^{-3} \mathrm{~W} \cdot \mathrm{m}^{-2}\right)$ is required; radiant flux density approximately 10 fold weaker was not effective [23]. Our direct experiments with white light (10,000 lux) applied to the skull indicate that photon energies move across this impediment through cerebral tissue and are emitted distally [24]. The slow latency for photon detection (1.7 s along the rostral-caudal axis; $0.7 \mathrm{~s}$ across the width of the skulls) compared to the "instantaneous" detection expected by direct light suggested the role of Grotthuss-like mechanisms involving protons.

Within the darkness of the internal organs and blood occupied by the virus the photon flux density is likely to be in the order of $10^{-12} \mathrm{~W} \cdot \mathrm{m}^{-2}$ [25]. This is consistent with the results from multiple studies [26] [27] that showed that cell-to-cell communication as well as functional electrical correlations involved power densities in this range [3]. Our experiments with specific filters have suggested that picoWatt per meter-squared photon patterns, if appropriately patterned, may be the "information" that initiates the much more glucose energy-demanding cascade of molecular pathways. We have shown this for preparations of microtubules [13]. In other words the inter-cell photon emissions and correlated information are equivalent to turning the ignition on or off in an automobile and involve minimal energy. The major energy that operates this method of conveyance is contained within the construction of the automobile.

In the balance of probabilities the static application of the optimal LED-emitting photons would not be as effective as the appropriate, physiologically-patterned pulsation of the light. The rationale for this statement is based upon what we have measured for weak, biofrequency magnetic fields. Different temporal patterns of weak (nanoTesla to microTesla) magnetic fields generated by specific point durations (the duration of each computer-generated voltage that generates the field) produce very specific effects [28] [29]. Light flashes coupled to magnetic fields applied across the brain enhance physiological effects [30]. Our recent unpublished results involving patterns of light flashes from near-ultraviolet and near-infrared LEDs applied to cancer cells have verified the efficacy of specific light wavelength patterns generated from exact point durations.

\section{Spectral Analysis of Cosic's RRM and Ebola Protein Patterns}

We have found that the greatest congruence between applied, physiologically-patterned magnetic fields, photon emissions from cells, and the responses of the molecular pathways of cells is not the absolute measures of the numbers of or flux density of photons over time, per se, but rather the spectral power densities of these changes. For example [31] the comparisons of the physiologically patterned (frequency- and phase-modulated) weak magnetic fields that slow the proliferation of cancer cells and the digitized patterns extracted from the quantitative electroencephalographic activity of a person with specific abilities to affect cancer cells showed no obvious visual similarities. However the spectral densities of the two patterns were significantly congruent.

The differences in correlation coefficients for protein sequences' spectral analyses were completed for the frequency patterns generated by the Cosic procedure for the proteins in Table 1. The proteins were sequenced according to their amino acids, analyzed by the Cosic method, and spectral analyzed using SPSS (SPSS-16 PC). The spectral profiles for each protein were compared by correlation between each pair of strains. Because raw spectral densities display an intrinsic decrease in power from the lowest to the highest frequencies, this serial order was covaried first before the cross-correlations were completed to minimize this possible artifact. However the changes in the strengths of coefficients were relatively minimal.

The results are shown in Table 3. The strongest correlations occurred between the two most lethal strains (Sudan-Zaire) compared to all of the other pairs of strain comparisons. In fact the strength of the averaged correlation coefficient according to one-way analysis of variance as a function of the six pairs was statistically significant $\left[\mathrm{F}(5,26)=8.85, \mathrm{p}<0.001\right.$; $^{\text {omega }}{ }^{2}=63 \%$ of variance explained $]$. The post hoc test $($ Tukey, $\mathrm{p}<0.05)$ indicated that the Sudan-Zaire comparison was significantly stronger than the other pairs that did not differ significantly from each other. One inference is that the Sudan-Zaire pair correlation strengths accommodated twothirds of the variability in all of the coefficients for the groups (pairs).

The most singularly powerful correlation $(r=0.62)$ occurred between the sGP spectral profiles for the Sudan and Zaire strains. These results suggest that all of these strains have the ability to attach, invade, and replicate inside the host cell. However the Zaire and Sudan strains are enhanced. The sGP component has been attributed to the capacity for the glycoprotein covering $(\sim 10 \mathrm{~nm})$ of the virus to fuse (and integrate) into the plasma cell membrane. This is followed by the insertion of the viral contents into the cell. At face value this enhanced correlation 
Table 3. Correlations between spectral densities of RRM profiles for different proteins for different pairs of Ebola strains.

\begin{tabular}{ccccccc}
\hline Protein & Sudan-Zaire & Sudan-Reston & Sudan-Ivory & Zaire -Reston & Zaire-Ivory & Ivory-Reston \\
\hline NP & 0.352 & 0.231 & 0.294 & 0.275 & 0.267 & 0.311 \\
VP35 & 0.407 & 0.349 & 0.298 & 0.216 & 0.265 & 0.375 \\
VP40 & - & - & - & - & - & - \\
GP1 & 0.366 & 0.315 & 0.258 & 0.246 & 0.23 & 0.309 \\
SGP & 0.624 & - & - & - & - & - \\
VP30 & 0.373 & 0.219 & 0.228 & 0.301 & 0.264 & 0.352 \\
VP24 & 0.514 & 0.206 & 0.196 & 0.283 & 0.312 & 0.344 \\
L & - & - & - & - & - & 0.395 \\
\hline
\end{tabular}

of spectral densities which only occurred between the two most lethal forms could be consistent with their high rates of successful modification of normal cells. It may be relevant that the associated energy per molecule from the Cosic frequency for the strains that generate the greatest mortality could exceed the born self-energy cost for an ion permeating a pure lipid bilayer for $\mathrm{H}_{3} \mathrm{O}^{+}$. This threshold is not reached for the energy from the Cosic frequencies for the non-lethal strains.

\section{Spectral Analyses Congruence with the Schumann Resonance}

During abiogenesis and the early production of amino acids from atmospheric gases through electrical discharges or "lightning" [32], the fundamental resonances of the earth were present [33]. The fundamental frequency which is determined by the ratio between the velocity of light and the earth's circumference is $\sim 7.8 \mathrm{~Hz}$ with harmonics that appear every $\sim 6 \mathrm{~Hz}$ (e.g., $14 \mathrm{~Hz}, 20 \mathrm{~Hz}, 26 \mathrm{~Hz}$ ). They are generated by the approximately 40 to 100 lighting discharges per second globally that originate primarily from equatorial regions.

Koenig [34] noted the conspicuous similarity between the structures of these resonances and human electroencephalographic patterns almost 50 years ago. The intensity of the magnetic field component of the fundamental frequency is about 2 to $4 \mathrm{nT}$ while the electric field component is about $1 \mathrm{mV} \cdot \mathrm{m}^{-2}$ [35]. These values are within the same order of magnitude and even approach the coefficients for the primary magnetic and electric field components associated with human cerebral activity [36].

Alterations in the amplitudes of the Schumann resonances reflect the variations in global thunderstorm activity and exhibit a yearly maximum during May and a minimum in October-November. There are intrinsic periodicities of 5, 10 and 20 days. The mild shift in frequency with a peak around $15 \mathrm{hr}$ UT has been attributed to the meridian drift in global lightning activity [35]. Increased amplitudes within the third and fourth harmonic precede some seismic events. What may be particularly relevant for biological processes is that the $125 \mathrm{~ms}$ cycles for completion of the circular waves display phase shifts approaching 20 to $25 \mathrm{~ms}$, which is considered to be one of the latencies required to add a base to a DNA sequence.

That very weak magnetic fields such as those generated normally between the earth surface and the ionosphere due to global lighting can show cross-spectral congruence with electroencephalographic activity within the human brain was recently reported by Saroka and Persinger [37]. Although the intensities may be considered "too weak", both quantitative calculations and direct comparisons in real time of rates of change in electroencephalographic power density within Schumann frequencies and actual power directly measured from Saroka's Sudbury station exhibit clear phase coherence.

To discern if there was spectral density congruence between the Cosic solutions for various components of the Ebola protein and the Schumann pattern, the two were correlated. Two random samples of Schumann resonances were obtained from an Italian station and our local (Saroka) station. Results for the Italian station are shown in Table 4. The Schumann spectral density correlation was strongest and statistically significant with the GP1 protein. This protein is associated with the $10 \mathrm{~nm}$ "spikes" that protrude from the major mass and allow the virus to anchor to the host's cell membrane. Hence if the most lethal forms whose SGPs are highly correlated were more "cohesive" because of the enhanced properties of the GP1 whose spectrum is correlated with the Schumann resonance, the probability of transcellular infection could be markedly enhanced. 
Table 4. Correlation coefficients between spectra densities of RRM Profiles for different proteins from Ebola and schumann resonance spectral densities. Only statistically significant $(\mathrm{p}<0.05)$ values are shown.

\begin{tabular}{cc}
\hline Protein & Schumann \\
NP & - \\
VP35 & - \\
VP40 & - \\
GP1 & 0.411 \\
SGP & - \\
VP30 & - \\
VP24 & 0.264 \\
L & - \\
\hline
\end{tabular}

For the Saroka (Sudbury) Station the only statistically significant cross-correlation again occurred for the GP1 protein (0.287). The congruence for GP1 was evident for both Schumann patterns separated by two loci (Canada and Italy), indicating although not proving a potential source of shared variance. This suggests that global variables that produce increases in the Schumann intensities which could involve uninvestigated stimuli such as the enhanced lightning (thunderstorm) frequencies associated with global warming or alterations in vertical atmospheric current density $\left(\sim 10^{-12} \mathrm{~A} \cdot \mathrm{m}^{-2}\right)$ from specific arrays of human population density could facilitate this activation [37]. We cannot exclude the possibility that man-made technical energies penetrating in the earth-ionosphere cavity could also modify Schumann factors.

\section{Conclusion}

Although current models for viral proliferation and contagion are congruent with accepted mechanisms, there may be parallel perspectives that could facilitate the understanding and treatment, particularly for the very lethal viruses such as Ebola. The transformation of amino acid sequences to spectral densities based upon de-localized electron densities as proposed by Irena Cosic completely differentiated the very lethal and effectively asymptomatic strains of Ebola. The electromagnetic wavelengths within the near ultraviolet for the lethal forms and the near infrared for the non-lethal forms indicate that application of the appropriately patterned "monochromatic" or narrow band, LED generated wavelengths might attenuate the undesirable activities that lead to mortality. The technique would be non-invasive, relatively inexpensive, and if successful would support the alternative model that molecular reactions can be simulated or virtually controlled by the equivalent electromagnetic energy applied as specific quanta of photons.

\section{Acknowledgements}

We thank Dr. W. E. Bosarge, Jr., CEO Capital Technologies, Inc. for his support of research that emphasizes conceptually different and innovative technologies. Special thanks to Dr. Blake T. Dotta for technical advice and contributions and to Professor Kevin Saroka for his Schumann data.

\section{References}

[1] Lee, J.E., Fusco, M.L., Oswald, W.B., Hessell, A.J., Burton, D.R. and Saphire, E.O. (2008) Structure of the Ebola Virus Glycoprotein Bound to an Antibody from a Human Survivor. Nature, 454, 177-182. http://dx.doi.org/10.1038/nature07082

[2] Licata, J.M., Johnson, R.F., Han, Z. and Harty, R.N. (2004) Contributions of Ebola Virus Glycoprotein, Nucleoprotein and VP24 to Budding of VP40 Virus-Like Particles. Journal of Virology, 78, 7344-7351. http://dx.doi.org/10.1128/JVI.78.14.7344-7351.2004

[3] Dotta, B.T., Saroka, K.S. and Persinger, M.A. (2012) Increased Photon Emission from the Head While Imagining Light in the Dark Is Correlated with Changes in Electroencephalographic Power: Support for Bokkon's Biophoton 
Hypothesis. Neuroscience Letters, 513, 151-154. http://dx.doi.org/10.1016/j.neulet.2012.02.021

[4] Persinger, M.A. (2010) $10^{-20}$ Joules as a Neuromolecular Quantum in Medicinal Chemistry: An Alternative Approach to Myriad Molecular Pathways. Current Medicinal Chemistry, 17, 3094-3098. http://dx.doi.org/10.2174/092986710791959701

[5] Cosic, I. (1994) Macromolecular Bioactivity: Is It Resonant Interaction between Macromolcules? IEEE Transactions of Biomedical Engineering, 41, 1101-1114. http://dx.doi.org/10.1109/10.335859

[6] Dotta, B.T., Murugan, N.J., Karbowski, L.M., Lafrenie, R.M. and Persinger, M.A. (2014) Shifting the Wavelengths of Ultraweak Photon Emissions from Dying Melanoma Cells: Their Chemical Enhancement and Blocking Are Predicted by Cosic's Theory of Resonant Recognition Model for Macromolecules. Naturwissenschaften, 101, 87-94. http://dx.doi.org/10.1007/s00114-013-1133-3

[7] Wu, H.-P.P. and Persinger, M.A. (2011) Increased Mobility and Stem-Cell Proliferation Rate in Dugesia tigrina Induced by $880 \mathrm{~nm}$ Light Emitting Diode. Journal of Photochemistry and Photobiology B: Biology, 102, 156-160. http://dx.doi.org/10.1016/j.jphotobiol.2010.11.003

[8] Trushin, M.V. (2004) Light-Mediated “Conversation” among Microorganisms. Microbiological Research, 159, 1-10. http://dx.doi.org/10.1016/j.micres.2003.11.001

[9] Popp, F.-A., Li, K.H., Mei, W.P., Galle, M. and Neuohr, R. (1988) Physical Aspects of Biophotons. Experientia, 44, 576-585. http://dx.doi.org/10.1007/BF01953305

[10] Fels, D. (2009) Cellular Communication through Light. PloS ONE, 4. http://dx.doi.org/10.1371/journal.pone.0005086

[11] Dotta, B.T. and Persinger, M.A. (2012) "Doubling” of Local Photon Emissions When Two Simultaneous, SpatiallySeparated, Chemiluminescent Reactions Share the Same Magnetic Field Configurations. Journal of Biophysical Chemistry, 3, 72-80. http://dx.doi.org/10.4236/jbpc.2012.31009

[12] Dotta, B.T., Murugan, N.M., Karbowski, L.M. and Persinger, M.A. (2013) Excessive Correlated Shifts in pH within Distal Solutions Sharing Phase-Uncoupled Angular Accelerating Magnetic Fields: Macro-Entangelment and Information Transfer. International Journal of Physical Sciences, 8, 1783-1787.

[13] Dotta, B.T., Vares, D.A.E., Buckner, C.A., Lafrenie, R.M. and Persinger, M.A. (2014) Magnetic Field Configurations Corresponding to Electric Field Patterns that Evoke Long-Term Potentiation Shift Power Spectra of Light Emissions from Microtubules from Non-Neural Cells. Open Journal of Biophysics, 4, 112-118. http://dx.doi.org/10.4236/ojbiphy.2014.44013

[14] Dotta, B.T., Lafrenie, R.M., Karbowski, L.M. and Persinger, M.A. (2014) Photon Emission from Melanoma Cells during Brief Stimulation by Patterned Magnetic Fields: Is It the Source Coupled to Rotational Diffusion within the Membrane? General Physiology and Biophysics, 33, 63-73. http://dx.doi.org/10.4149/gpb 2013066

[15] Kobayashi, K., Okabe, H., Kawano, S., Hidaka, Y. and Hara, K. (2014) Biophoton Emission Induced by Heat Shock. PLOS ONE, 9.

[16] Fitzgerald, M., Bartlett, C.A., Payne, S.C., Hart, N.S., Rodger, J., Harvey, A.R. and Dunlop, S.A. (2010) Near Infrared Light Reduces Oxidative Stress and Preserves Function in CNS Tissue Vulnerable to Secondary Degeneration Following Partial Transection of the Optic Nerve. Journal of Neurotrauma, 27, 2107-2119.

http://dx.doi.org/10.1089/neu.2010.1426

[17] de Souza, S.C., Munin, E., Alves, L.P., Salgado, M.A.C. and Pacheco, M.T.T. (2005) Low Power Laser Radiation at $685 \mathrm{~nm}$ Stimulates Stem-Cell Proliferation Rate in Dugesia tigrina during Regeneration. Journal of Photochemistry and Photobiology, 80, 203-207. http://dx.doi.org/10.1016/j.jphotobiol.2005.05.002

[18] Mitsunaga, M., Ogawa, M., Kosaka, N., Rosenblum, L.T., Choyke, P.L. and Kobayashi, H. (2011) Cancer Cell-Selective in Vivo near Infrared Photoimmunotherapy Targeting Specific Membrane Molecules. Nature Medicine, 17, 1685-1691. http://dx.doi.org/10.1038/nm.2554

[19] Dotta, B.T., Buckner, C.A., Cameron, D., Lafrenie, R.M. and Persinger, M.A. (2011) Biophoton Emissions from Cell Cultures: Biochemical Evidence for the Plasma Membrane as the Primary Source. General Physiology and Biophysics, 30, 301-309.

[20] Nissila, J., Manttari, S., Sarkija, T., Tuominen, H., Takala, T., Timonen, M. and Saarela, S. (2012) Encephalopsin (OPN3) Protein Abundance in the Adult Mouse Brain. Journal of Comparative Physiology A, 198, 833-839.

[21] Starck, T., Nisslia, J., Aunio, A., Abou-Elseoud, A., Remes, J., Nikkinen, J., Timonen, M., Takala, T., Tervonen, O. and Kiviniemi, V. (2012) Stimulating brain tissue with bright light alters functional connectivity in brain at resting state. World Journal of Neuroscience, 2, 81-90.

[22] Lubsandorzhiev, B.K., Pokhil, P.G., Vasilev, R.V. and Vyatchin, Y.E. (2003) Measurements of Group Velocity of Light in the Lake Baikai Water. Nuclear Instruments and Methods in Physics Research Section A, 502, 168-171. http://dx.doi.org/10.1016/S0168-9002(03)00269-9 
[23] Rosenthal, N.E., Sack, D.A. and Wehr, T.A. (1987) Light, Seasonal Effects on Mood. In: Adelman, G., Ed., Encyclopedia of Neuroscience, Birkhauser, Boston, 586-588.

[24] Persinger, M.A., Dotta, B.T. and Saroka, K.S. (2013) Bright Light Transmits through the Brain: Measurement of Photon Emissions and Frequency-Dependent Modulation of Spectral Electroencephalographic Power. World Journal of Neuroscience, 3, 10-16. http://dx.doi.org/10.4236/wjns.2013.31002

[25] Kobayashi, M., Takeda, M., Sato, T., Yamazaki, Y., Kaneko, K., Ito, K.I., Kato, H. and Inaba, H. (1999) In Vivo Imaging of Spontaneous Ultraweak Photon Emission from a Rat's Brain Correlated with Cerebral Energy Metabolism and Oxidative Stress. Neuroscience Research, 34, 103-113. http://dx.doi.org/10.1016/S0168-0102(99)00040-1

[26] Yoon, Y.Z., Kim, J., Lee, B.C., Kim, Y.U., Lee, S.K. and Soh, K.S. (2005) Changes in Ultraweak Photon Emission and Heart Rate Variability of Epinephrine-Injected Rats. General Physiology and Biophysics, 24, 147-159.

[27] Vogel, R. and Suessmuth, R. (1998) Interaction of Bacterial Cells with Weak Light Emission from Cell Media. Bioelectrochemistry and Bioenergetics, 45, 93-101. http://dx.doi.org/10.1016/S0302-4598(98)00067-1

[28] Martin, L.J., Koren, S.A. and Persinger, M.A. (2004) Thermal Analgesic Effects from Weak, Complex Magnetic Fields and Pharmacological Interactions. Pharmacology Biochemistry and Behavior, 78, 217-227. http://dx.doi.org/10.1016/j.pbb.2004.03.016

[29] Mach, Q.H. and Persinger, M.A. (2009) Behavioral Changes with Brief Exposures to Weak Magnetic Fields Patterned to Simulate Long-Term Potentiation. Brain Research, 1261, 45-53. http://dx.doi.org/10.1016/j.brainres.2009.01.002

[30] De Sano, C.F. and Persinger, M.A. (1987) Geophysical Variables and Behavior: XXXIX. Alterations in Imaginings and Suggestibility during Brief Magnetic Field Exposures. Perceptual and Motor Skills, 64, 968-970. http://dx.doi.org/10.2466/pms.1987.64.3.968

[31] Karbowski, L.M., Harribance, S.L., Buckner, C.A., Mulligan, B.P., Koren, S.A., Lafrenie, R.M. and Persinger, M.A. (2012) Digitized Quantitative Electroencephalographic Patterns Applied as Magnetic Fields Inhibit Melanoma Cell Proliferation in Culture. Neuroscience Letters, 523, 131-134. http://dx.doi.org/10.1016/j.neulet.2012.06.059

[32] Johnson, A.P., Cleaves, H.J., Dworkin, J.P., Glavin, D.P., Lazcano, A. and Bada, J.L. (2008) The Miller Volcanic Spark Discharge Experiment. Science, 322, 404. http://dx.doi.org/10.1126/science.1161527

[33] Graf, F.E. and Cole, E.R. (1974) Precambrian ELF and Abiogenesis. In: Persinger, M.A., Ed., ELF and VLF Electromagnetic Field Effects, Praeger, New York, 243-275.

[34] Koenig, H.L., Krueger, A.P., Lang, S. and Sonning, W. (1981) Biological Effects of Environmental Electromagnetism. Springer-Verlag, New York. http://dx.doi.org/10.1007/978-1-4612-5859-9

[35] Persinger, M.A. (2014) Schumann Resonance Frequencies Found within Quantitative Electroencephalographic Activity: Implications for Earth-Brain Interactions. International Letters of Chemistry, Physics and Astronomy, 11, 24-32.

[36] Nickolaenko, A. and Hayakawa, M. (2014) Schumann Resonance for Tyros. Springer, Tokyo. http://dx.doi.org/10.1007/978-4-431-54358-9

[37] Sarorka, K.S. and Persinger, M.A. (2014) Quantitative Evidence for Direct Effects between Earth-Ionosphere Schumann Resonances and Human Cerebral Cortical Activity. International Letters of Chemistry, Physics and Astronomy, 20, 166-194. 
Scientific Research Publishing (SCIRP) is one of the largest Open Access journal publishers. It is currently publishing more than 200 open access, online, peer-reviewed journals covering a wide range of academic disciplines. SCIRP serves the worldwide academic communities and contributes to the progress and application of science with its publication.

Other selected journals from SCIRP are listed as below. Submit your manuscript to us via either submit@scirp.org or Online Submission Portal.
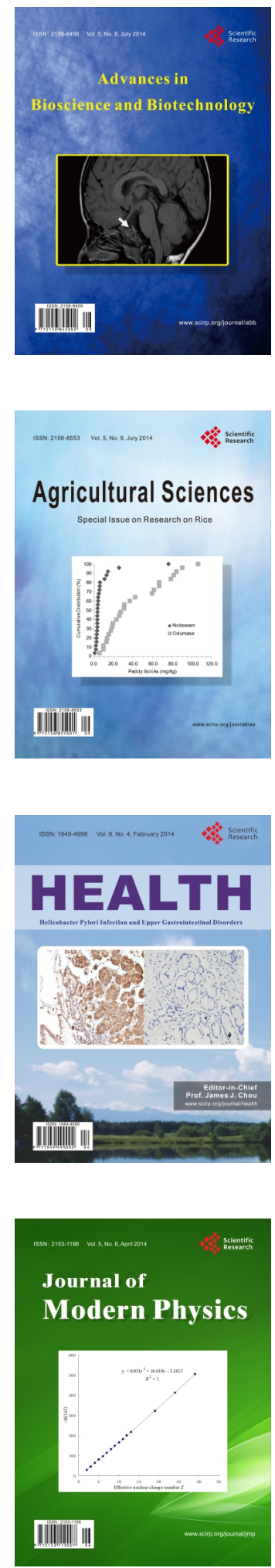
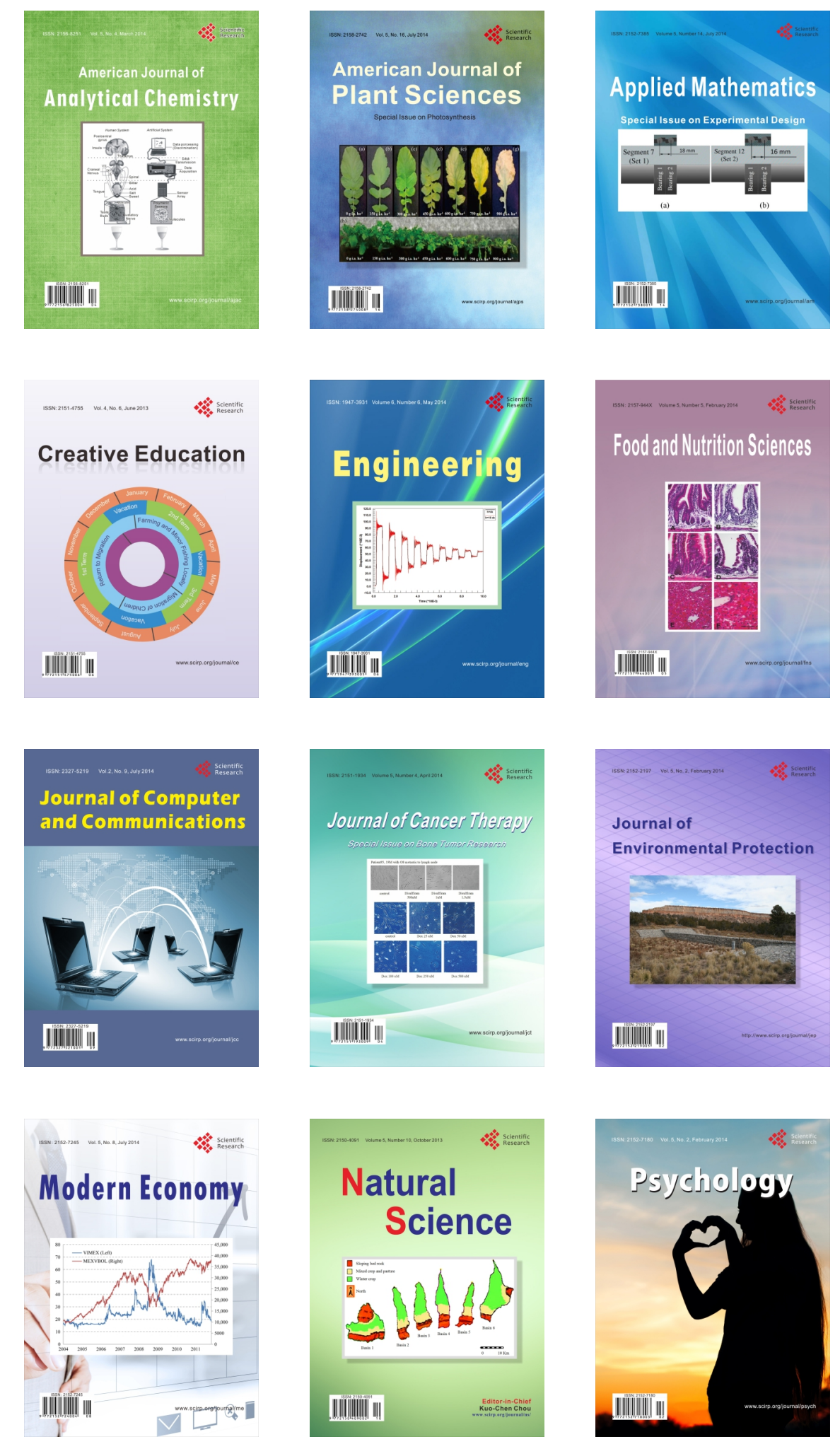